\title{
The Bjorken Limit in a Simple Model of Electron-Nucleon Inelastic Scattering.
}

A. P. Contogouris

Department of Physics, McGill University - Montreal

(Lett. Nuovo Cimento, 4, $1213(1970)$ )

The Figures 2 and 3 of this paper should be interchanged.

(C) by Società Italiana di Fisica

Proprietà letteraria riservata

Direttore responsabili: GIULIANO TORALDO DI FRANCIA

Stampato in Bologaa dalla Tipografia Compositori coi tipi della Tipografia Monograf

Questo fassoicolo è stato licenziato dai torchi il 17-III-1971 